\title{
The Development of GPAS 5 Steps Teaching Model for Enhancing Learning Skills in Thailand 4.0 Era
}

\author{
ChanasithSithsungnoen ${ }^{1}$, Wisud Po-Ngern $^{2}$, AkkachaiPoomraruen $^{3}$, Maream Nillapun ${ }^{4}$ \\ 1,2,3,4 Faculty of Education Silpakorn University, NakornPathom, Thailand \\ 1 sithchon@hotmail.com
}

\begin{abstract}
The purposes of this research were 1) to develop GPAS 5Steps Teaching Model for enhancing learning skills in Thailand 4.0 era, 2) to evaluate the effectiveness ofconducted model in aspects of 2.1) instructors: 2.1.1) knowledge and understanding about ofinstruction, 2.1.2) instructional design ability, 2.1.3) instructional ability, 2.1.4) instructional innovation, and 2.1.5) instructors' opinion towards the model, 2.2) students: 2.2.1) higher order thinking, 2.2.2) innovative skills, 2.2.3) innovative construction skills, 2.2.4) morality and ethics, and 2.2.5) opinion towards instruction, and 3) to disseminate using theconducted model, to childhood, secondary and elementary education. The subjects consisted of school administrators, instructors, and students of Satit Vajiralongkorn Rajabhat University School in the academic year 2018. The instruments used included 1) GPAS 5Steps Teaching Model, 2) test, 3) instructors and learners' ability evaluation, 4) observation form, and 5) opinion evaluation. The statistical analysis utilized mean, percentage, standard deviation, and content analysis.

The results were as follows: 1) GPAS 5Steps Teaching Model included principle, objectives, instructions, assessment and evaluation, and success factors. 2) The effectiveness of the conducted model was indicated by:2.1) instructors 2.1.1) instructors' knowledge at the highest level, 2.1.2) design ability was at a high level, 2.1.3) teaching abilities were at the highest levels, 2.1.4) innovative abilities were at the highest levels, 2.1.5) instructors' opinions toward the conducted model benefited both instructors and students, 2.2) students 2.2.1) students' higher order thinking were at a high level, 2.2.2) innovative skills were at high level, 2.2.3) innovative construction skills were at a high level, 2.2.4) morality and ethics were at high levels, and 2.2.5) opinion toward instructions were at a high level. 3) In conclusion, the disseminatedresults provided benefit to instructors as pedagogues and the overall student learning experience.
\end{abstract}

Keywords

teaching model, GPAS 5 Steps instruction, learning skills in Thailand 4.0 era

Article Received: 10 August 2020, Revised: 25 October 2020, Accepted: 18 November 2020

\section{Introduction}

In contemporary society, education seeks to equip students with the skills and attributes to navigate and succeed in a challenging period of rapid cultural, societal, economic and technological change. 3R 3Cs are learning skills for preparing learners to develop emotionally and cognitively in society, exemplified by the basic'3R's - reading, writing, arithmetic and the' $3 \mathrm{Cs}$ ' - critical thinking and problem solving, creativity and innovation, cross-cultural understanding, all essential forcollaborative teamwork and leadership, communication, information and media literacy, computing and ICT literacy, career and learning skills and compassion (Office of the Education Council: ONEC, 2018: introduction). Moreover, the Minister of Education for Thailand recentlyannounced the use of Basic Education Core Curriculum B.E. 2551 (A.D. 2008) to promote independent learning skills within the school framework. This scheme is expected not only to create more technologically and cognitively astute learners but make the learning process a more emotionally sustaining and positive experience on the journey to collaborative citizenship.(Thailand Minister of Education, 2008: 17). Essential components of this initiative for contemporary and future students is the need for creativity, innovation, and cross-cultural understanding(Thailand Minister of Education, 2019: 4-6).In short, learnersmust be able to reach the digital world, create innovation, have creativity, and develop innovation. Furthermore, the promotion and encouragement of life skills and analytical thinking by providing active learning in theclassroom, must work alongside digital literacies.(Thailand Minister of Education B.E. 2562(2019)).

According to the educational position report 2016/2017 guideline of educational reform into Thailand 4.0 era (Office of the Education Council, 2018) teacher professional development is a crucialfactor for improving basic education quality.This Report suggests that education should focus onanalytical thinking alongside experimental and active learning instruction. A learner-centered curriculum, thereforeis neededto drive educational reform, an initiative which empowers students to embrace their differences from other students and use their own knowledge, learning aptitudes and motivations as tools for self development. This philosophy is consistent withthe royal educational thoughtof His Majesty King Bhumibol Adulyadej(King Rama IX) and The King Maha Vajiralongkorn (King Rama X)who both contend that lesson study and the Professional Learning Community- PLC -are essential for role modeling the effective teacher in the 21 st century.

PLC is used for professional development for teachers and administrators of government and private sections in order to gain knowledge and better understand student-centered instruction.Performing, monitoring, and supervising are systematic driversfor educational reform, focusing on professional improvement and school-based learning- SBT for teachers to study and create innovation from classroom situations.

GPAS 5 Steps instructional process of theInstitute of Academic Development- IAD (2018) is a process which emphasizesreflective self-knowledge as a construction for student learning. The processes begin byidentifying 
taskswhich allow students to summarize their understanding into knowledge. Then, students must work on and assimilate, the relationship of concept and theory into their self-knowledge. Thus, self-knowledge and construction processes are built through learning activities - data collection is provided for students to seek and choosecritical information to collect as their sources and for data categorization. Students subsequently used collected data for problem solving activities. An essential part of the process is assessment and evaluation: students are encouraged to check, review and ask questions of peers related to data findings and teaching performance. (SiriwanVanichwatanavorachai, 2016: 151-152).

As noted above, the Thai government currently emphasizes educational reform to galvanize, improve and encourage the generality of Thai learners in the 4.0 era. Arguably, an essential strand of this overall reconsidering of Thai education is the ability of students to take more control of their own learning: GPAS instruction, therefore, is deemed essential to improve students' higher order thinking and analytical thinking skills:in an increasingly culturally diverse and technologically reliant global economy and society, independent thinking processes, as part of life long learning, will become crucial for Thailand's own cultural and economic growth. The GPAS model, therefore assumes even greater national significance, in particular the need to develop consistent strategies for developing educational instructors.

Thus, the Faculty of Education ofSilpakorn University, Satit Vajiralongkorn Rajabhat University School, and the Institute of Academic Development- IAD are in collaboration to make the Memorandum Of Understand (MOU) to develop academic research and innovation toenhance instructors' pedagogic abilities.

The development is a response to the educational reform of the national standard B.E. 2561 (A.D. 2018)- Desired Outcomes of Education, DOE Thailand, underpinned by morality,social and people skills, and social value knowledge. The 20-Year National Strategy (2017-2036), National Education Plan 2017-2036, and Basic Education Core Curriculum B.E. 2551 (edition B.E. 2560) are focused on educational reform which support life long learning; 3R and 3Cs emphasize morality and ethics based on Sufficiency Economy principles - to nurture social value and social empathy, qualities essential to construct an innovative Thai society of 4.0 era. Consequently, the model is conducted in early childhood, primary, and secondary education of Satit Vajiralongkorn Rajabhat University School.

\section{Research objectives}

1. Todevelop GPAS 5Steps Teaching Model for enhancing learning skills in Thailand 4.0 erafor early childhood, primary, and secondary education.

2. To evaluate the effectiveness of GPAS 5Steps Teaching Model for enhancing leaning skills in Thailand 4.0 erafor early childhood, primary, and secondary education in aspects

2.1. Instructors: 1) knowledge and understanding about GPAS 5Steps instruction, 2) instructional design ability based on GPAS 5Steps, 3) instructional ability based on
GPAS 5Steps, 4) instructional innovation, and 5) opinion towards GPAS 5Steps Teaching Model

2.2. Students: 1) higher order thinking ability, 2) innovative skills, 3) innovative construction skills, 4) morality and ethics, and 5) opinion towards GPAS 5 Steps instruction.

3. To disseminate using GPAS 5Steps Teaching Model for enhancing leaning skills in Thailand 4.0 erafor early childhood, primary, and secondary education.

\section{Research Hypothesis}

1. Instructors:

1.1. Knowledge and understanding of GPAS 5Steps instruction are at a high level.

1.2. Instructional design ability based on GPAS 5 Steps are at high level.

1.3. Instructional ability based on GPAS 5 Steps are at high level.

1.4. Instructional innovations are at high level.

2. Students:

2.1. Higher order thinking abilities are at a high level.

2.2. Innovative skills are at high level.

2.3. Innovative construction skills are at high level.

2.4. Morality and ethics are at high level.

\section{Research Scopes}

\section{Content}

GPAS 5Steps Teaching Model for enhancing learning skills in Thailand 4.0 erafor early childhood, primary, and secondary educationof Satit Vajiralongkorn Rajabhat University School.

Purpose and informants

2.1. Developmental processes of GPAS 5 Steps Teaching Model for Enhancing Learning Skills in Thailand 4.0 Era

1) 85 school administrators and teachersof Satit Vajiralongkorn Rajabhat University School.

2) $\quad 1,830$ students of Satit Vajiralongkorn Rajabhat University School included 2.1) 197 early childhood students, 2.2) 708 primary students, and 2.3) 925 secondary students.

2.2. Evaluation processes of GPAS 5Steps Teaching Model for enhancing leaning skills in Thailand 4.0 era were evaluated by 9 experts and 48 school administrators and teachers from early childhood, primary, and secondary education of 4 schools included Satit schools,schools inOffice of the Basic Education Commission: OBEC, municipal Schools, andpublic schools at total 14 schools; 5 people each school.

2.3 Results of the GPAS 5Steps Teaching Model for enhancing leaning skills in Thailand 4.0 era were disseminated usingearly childhood, primary, and secondary education from 4schools including2 Satis schools, schools in Office of the Basic Education Commission: OBEC, municipal Schools, andpublic schools. 14 schools in central, 19 schools in Northeastern, and 12 schools in Southern region included228 school administrators and instructors, 2,880 students, and 15 supervisors. 


\section{Research methodology}

\section{Phase 1 need assessment analysis: na}

Basic information, educational principles, 21st century learning skills and theory, professional learning community, supervising and coaching, and GPAS 5 Steps instruction were studied as need analysis.

\section{Phase 2 Design \& Development: D}

Depth interview were used with school administrators, heads of 8 subject areas from early childhood, primary, and secondary education of Satit Vajiralongkorn Rajabhat University School for finding principles, ideas, procedure, assessment and evaluation, condition or factor of GPAS 5 Steps teaching model.

\section{Phase 3 Implementation: I}

GPAS 5 Steps teaching model for enhancing learning skills in Thailand 4.0 era was used with instructors and students of early childhood, primary, and secondary education.

\section{Phase 4 Evaluation: E}

1) The test was provided to evaluate knowledge and understanding about GPAS 5 Steps teaching model, instructional design ability,instructional ability, instructional innovation, and instructors' opinion towards GPAS 5 Steps teaching model for enhancing learning skills in Thailand 4.0 era. The test provided to 85 instructors of Satit Vajiralongkorn Rajabhat University School from early childhood, primary, and secondary education.

2) The evaluation forms were used to evaluate students' higher order thinking ability, innovative skills, innovative construction skills, moralityand ethics, and opinion towards GPAS 5 Steps instruction.The result from instructor evaluation based on GPAS 5 Steps and learning achievement analysis were used to evaluate as well.

\section{Phase 5 Improvement: I}

The 9 expertsand 48 school administrators and instructors from 4 school sectionsjoined the connoisseurship in order to improve teaching model and manual.

\section{Phase 6 Dissemination: D}

Dissemination of using conducted model to subject (purposive selection) in early childhood, primary, and secondary education from all 4 sections; 2 schools from Office of the Basic Education Commission: OBEC, municipal Schools, andpublic schools at 4 religions.

\section{Research results}

Part 1: The development of GPAS 5 Steps exalted teaching model for enhancing learning skills in Thailand 4.0 Era included the following components:

1. Principle:For exalting instruction quality in 4.0 era and 21 st century learning skills, especially to enhance the creative innovation of students, thus GPAS 5 Steps instruction are used via professional learning community: PLC.

2. Objectives:

2.1 Instructors: 1) should be able to understand active learning via the GPAS 5

Steps concept, 2) are able to create and design active learning via GPAS 5 Steps, 3) are able to provide learning activity by using active learning via GPAS 5 Steps, and 4) are able to create instructional innovation.

2.2 Students: 1) are able to improve higher order thinking, 2) to be the

innovators, 3) are able to create innovation, and 4) have morality and ethics.

3. GPAS 5 Steps instructional procedures:1) gathering information, 2) processing, 3) applying and construct the knowledge, 4) applying the communication skill, and 5) self-regulating.

4. Exalted educational quality procedures:

4.1 Setting vision and goal: school administrators, instructors, coaches, and experts

plan collaboratively to set vision, goal, and mission for professional development via GPAS 5 Steps instruction in order to enhance students' 21st century learning skills. Knowledge and understanding of GPAS 5 Steps instruction, instructional innovation, design ability, and effective learning activities were essential skills needed for instructors.

4.2 Knowledge sharing: was used as processes for coaches and experts in sharing

information and data about GPAS 5 Steps, and also provided example of instructional innovation, technology materials, and assessment and evaluation that emphasized teaching skills, performance and morality.

4.3 Coach and supervision: were used as a guideline for coaches and experts plan

collaboratively about ideas, model, procedure, instruments, activities evaluation, and supervision using GPAS 5 Steps instruction. The supervisory plan covered topics of lesson planning, instructional innovation, coaching, teaching, observing both via online and face-to-face, and after-action review (AAR) to develop learning outcome, innovation, higher order thinking, and morality of students.

4.4 Lesson study: was used by school administrators, instructors, coaches, and experts

for creating online PLC of each subject learning area. The PLC processes were used both online and face-to-face for sharing, supervising, coaching, classroom observing, and monitoring. The classroom observation, reflection of instructor and students' behavior wereset as an observation plan, which included 3 sub-activities: 1) plan and design lesson, 2) implement and observe, and 3) review and refine. 4.5 Evaluate the expected results : evaluate the data, school administrators, instructors, 
coaches, and experts collaboratively gauged instructors' knowledge and understanding of GPAS 5 Steps, as well as instructional ability, instructional innovation, materials, assessments and evaluation, and suggestions. The students' higher order thinking, innovative skills, innovative construction ability, and morality and ethic were also estimated and appraised.
5. Assessment and evaluation: authentic assessment and assessment for learning

6. Factors for success: 1) empowering, 2) technology literacy, 3) expert coaching, and 4)

knowledge and understanding about GPAS 5 Steps instruction.

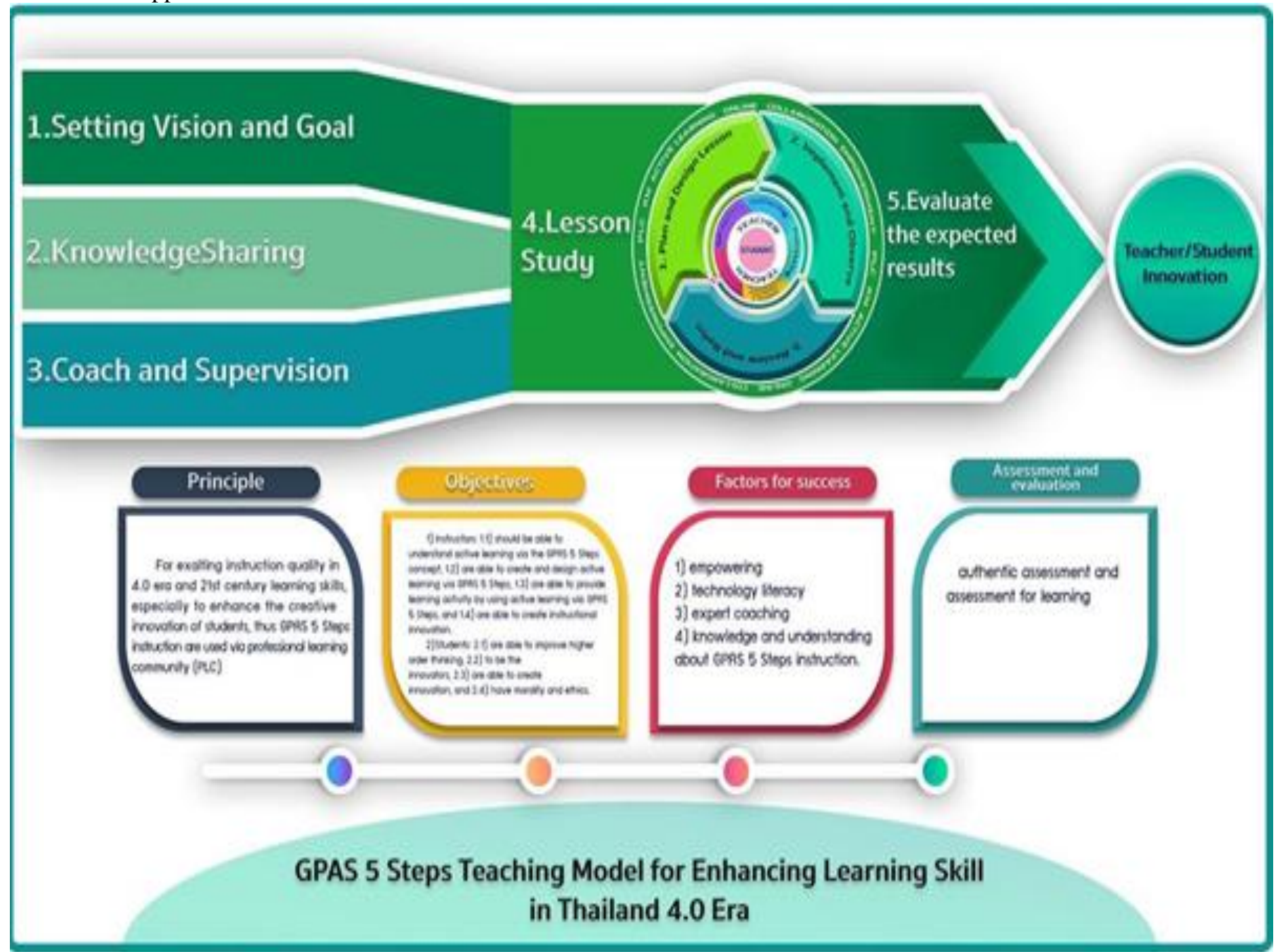

Figure: GPAS 5 Steps Teaching Model for Enhancing Learning Skills in Thailand 4.0 Era

Part 2: The effectiveness of using GPAS 5 Steps teaching model for enhancing learning skills in Thailand 4.0 era in early childhood, primary, and secondary education

1. Early childhood, primary, and secondary instructors' knowledge and understanding about GPAS 5 Steps were at the highest level, 44.71 percent were at high level, 43.53 percent were at medium level, and 11.77 percent were at a fair level.

Table 1 showsinstructors' knowledge and understanding of GPAS 5 Steps instruction

\begin{tabular}{|c|c|c|c|c|c|c|c|c|c|}
\hline \multirow{3}{*}{$\begin{array}{c}\text { Score range } \\
\text { (total } 30 \\
\text { points) }\end{array}$} & \multicolumn{6}{|c|}{ Educational level } & \multirow{2}{*}{\multicolumn{2}{|c|}{ total }} & \multirow{3}{*}{$\begin{array}{c}\text { Quality } \\
\text { level }\end{array}$} \\
\hline & \multicolumn{2}{|c|}{ Early childhood } & \multicolumn{2}{|c|}{ primary } & \multicolumn{2}{|c|}{ secondary } & & & \\
\hline & amount & $\%$ & amount & $\%$ & amount & $\%$ & amount & $\%$ & \\
\hline $26-30$ & 6 & $\begin{array}{c}66.67 \\
(1)\end{array}$ & 11 & $\begin{array}{c}37.94 \\
(2)\end{array}$ & 21 & $\begin{array}{c}44.68 \\
(1)\end{array}$ & 38 & $\begin{array}{c}44.71 \\
\text { (1) }\end{array}$ & highest \\
\hline $21-25$ & 2 & $\begin{array}{c}22.23 \\
(2)\end{array}$ & 17 & $\begin{array}{c}58.62 \\
(1)\end{array}$ & 18 & $\begin{array}{c}38.30 \\
(2)\end{array}$ & 37 & $\begin{array}{c}43.53 \\
(2)\end{array}$ & high \\
\hline $15-20$ & 1 & $\begin{array}{c}11.12 \\
(3)\end{array}$ & 1 & $\begin{array}{c}11.12 \\
(3)\end{array}$ & 8 & $\begin{array}{c}17.03 \\
(3)\end{array}$ & 10 & $\begin{array}{c}11.77 \\
(3)\end{array}$ & fair \\
\hline Total & 9 & 100 & 29 & 100 & 47 & 100 & 85 & 100 & \\
\hline
\end{tabular}

2. Early childhood, primary, and secondary instructors' design ability were at a high level $(=4.01$, S.D.= 0.43). Consideration of all aspects found that, 1 ; introduction of lesson plan, and 2; GPAS 5 Steps learning activities, both were at high level. Aspect 3; material and learning resources, were found that primaryinstructors had the highest GPAS 5 Steps instructional design ability, then secondary and early childhood. 
Table 2 showsinstructors' design ability based on GPAS 5 Steps

\begin{tabular}{|c|c|c|c|c|c|c|c|c|c|c|c|c|}
\hline \multirow[b]{3}{*}{ Item for evaluation } & \multicolumn{9}{|c|}{ Educational Level } & \multirow{2}{*}{\multicolumn{3}{|c|}{ Total }} \\
\hline & \multicolumn{3}{|c|}{ Early childhood } & \multicolumn{3}{|c|}{ primary } & \multicolumn{3}{|c|}{ secondary } & & & \\
\hline & $\bar{x}$ & S.D & 登可 & $\overline{\mathrm{x}}$ & S.D & 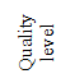 & $\overline{\mathrm{x}}$ & S.D & 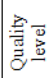 & $\overline{\mathrm{x}}$ & S.D & 造 \\
\hline $\begin{array}{l}\text { 1. Introduction of } \\
\text { lesson plan }\end{array}$ & $\begin{array}{l}3.62 \\
(3)\end{array}$ & 0.37 & high & $\begin{array}{l}4.72 \\
(1)\end{array}$ & 0.33 & highest & $\begin{array}{l}3.66 \\
(2)\end{array}$ & 0.39 & high & $\begin{array}{c}4.00 \\
(1)\end{array}$ & 0.36 & high \\
\hline $\begin{array}{l}\text { 2. Leaming activity } \\
\text { based onGPAS } 5 \\
\text { Steps }\end{array}$ & $\begin{array}{l}3.68 \\
(2)\end{array}$ & 0.49 & high & $\begin{array}{l}4.60 \\
(3)\end{array}$ & 0.51 & highest & $\begin{array}{c}3.74 \\
(1)\end{array}$ & 0.42 & high & $\begin{array}{l}4.00 \\
(1)\end{array}$ & 0.47 & high \\
\hline $\begin{array}{l}\text { 3.Leaming materials } \\
\text { and resources }\end{array}$ & $\begin{array}{l}3.71 \\
(1)\end{array}$ & 0.43 & high & $\begin{array}{l}4.64 \\
(2)\end{array}$ & 0.46 & highest & $\begin{array}{l}3.63 \\
(3)\end{array}$ & 0.44 & high & $\begin{array}{l}3.99 \\
(2)\end{array}$ & 0.44 & high \\
\hline Total & $\begin{array}{l}3.67 \\
(3)\end{array}$ & 0.43 & high & $\begin{array}{l}4.66 \\
(1)\end{array}$ & 0.44 & highest & $\begin{array}{l}3.68 \\
(2)\end{array}$ & 0.42 & high & 4.01 & 0.43 & high \\
\hline
\end{tabular}

Early childhood, primary, and secondary instructors' teaching ability were at the highest level; 69.42 percent. Consideration of each education level were found that secondary instructors' teaching ability were at the highest level; 72.34 percent, primarywere 68.96 percent, and early childhood were 55.56 percent.

Table 3 shows instructors' teaching ability based on GPAS 5 Steps

\begin{tabular}{|c|c|c|c|c|c|c|c|c|c|}
\hline \multirow{3}{*}{$\begin{array}{c}\text { Score range } \\
\text { (total 33 } \\
\text { points) }\end{array}$} & \multicolumn{6}{|c|}{ Educational Level } & \multirow{2}{*}{\multicolumn{2}{|c|}{ Total }} & \multirow{3}{*}{$\begin{array}{c}\text { Quality } \\
\text { level }\end{array}$} \\
\hline & \multicolumn{2}{|c|}{$\begin{array}{c}\text { Early } \\
\text { childhood }\end{array}$} & \multicolumn{2}{|c|}{ Primary } & \multicolumn{2}{|c|}{ Secondary } & & & \\
\hline & amount & $\%$ & amount & $\%$ & amount & $\%$ & amount & $\%$ & \\
\hline $28-33$ & 5 & $\begin{array}{c}55.56 \\
(3)\end{array}$ & 20 & $\begin{array}{c}68.9 \\
6 \\
(2) \\
\end{array}$ & 34 & $\begin{array}{c}72.3 \\
4 \\
(1) \\
\end{array}$ & 59 & $\begin{array}{c}69.42 \\
\text { (1) }\end{array}$ & highest \\
\hline $22-27$ & 4 & 44.45 & 9 & $\begin{array}{c}31.0 \\
4\end{array}$ & 13 & $\begin{array}{c}27.6 \\
6\end{array}$ & 26 & $\begin{array}{c}30.59 \\
\text { (2) }\end{array}$ & high \\
\hline total & 9 & 100 & 29 & 100 & 47 & 100 & 85 & 100 & - \\
\hline
\end{tabular}

3. Early childhood, primary, and secondary instructors' innovative ability were at the highest level mostly; 58.83, then 40 percent were at high level, and were at a fair level 1.18 percent. Consideration of each education level were found that secondary instructors had innovative ability were at the highest level; 72.34 percent, early childhood instructors were 55.56 percent, and primary instructors were 37.93 percent.

Table 4 shows instructors' innovative ability

\begin{tabular}{c|c|c|c|c|c|c|c|c|c}
\hline \multirow{2}{*}{$\begin{array}{c}\text { Score range } \\
\text { (total 30 } \\
\text { points) }\end{array}$} & $\begin{array}{c}|c| \\
\text { Early } \\
\text { childhood }\end{array}$ & \multicolumn{2}{|c|}{ Primary } & \multicolumn{2}{|c|}{ Secondary } & \multicolumn{2}{|c|}{ Total } & \multirow{2}{*}{$\begin{array}{c}\text { Quality } \\
\text { level }\end{array}$} \\
\cline { 2 - 10 } & amount & $\%$ & amount & $\%$ & amount & $\%$ & amount & $\%$ & \\
\hline $26-30$ & 5 & $\begin{array}{c}55.5 \\
6\end{array}$ & 11 & $\begin{array}{c}37.9 \\
(2)\end{array}$ & 34 & $\begin{array}{c}72.34 \\
(1)\end{array}$ & 50 & $\begin{array}{c}58.83 \\
(1)\end{array}$ & highest \\
\hline $16-25$ & 4 & $\begin{array}{c}44.4 \\
5\end{array}$ & 17 & $\begin{array}{c}58.6 \\
2\end{array}$ & 13 & 27.66 & 34 & $\begin{array}{c}40 \\
(2)\end{array}$ & high \\
\hline 15 and lower & - & - & 1 & 3.45 & - & - & 1 & $\begin{array}{c}1.18 \\
(3)\end{array}$ & fair \\
\hline Total & $\mathbf{9}$ & $\mathbf{1 0 0}$ & $\mathbf{2 9}$ & $\mathbf{1 0 0}$ & $\mathbf{4 7}$ & $\mathbf{1 0 0}$ & $\mathbf{8 5}$ & $\mathbf{1 0 0}$ & \\
\hline
\end{tabular}

4. Instructors' opinion toward GPAS 5 Steps teaching model for enhancing learning skills in Thailand 4.0 era found that 1) the conducted model provided benefit for instructors;for instance, instructors had schedule for working, systematic learning activities, and innovation which improved students' creativity and analytical thinking. 2) the conducted model provided benefit for students; for instance, students improved their analytical thinking, synthesis, creativity, systematical planning, collaborative working, problem solving, and joyful learning. 3) A number of issues adversely affected the model: inappropriate length of time for doing activities, unavailable technology, a lack of depth around learners' analysis, assessment, and construction innovative ability. 4) Instructors provided extra materials and technological resources based on inter-subject task integration to overcome these issues. This strategy enabled coach and school administratorsto stimulate, empower, and provide reward to instructors.

5. Early childhood, primary, and secondary students' higher order thinking were at a high level mostly, 44.76. Then, 38.80 percent of students were at the highest level, and 16.45 percent were at fair a level. Consideration of each education level found that early childhood students' higher order thinking were at the highest level; 47.21, then secondary students were 45.84 , and primary students were 42.66 percent.

Table 5 showsstudents' higher order thinking

\begin{tabular}{|c|c|c|c|c|c|c|c|c|c|}
\hline \multirow{3}{*}{$\begin{array}{c}\text { Score } \\
\text { range } \\
\text { (total } 16 \\
\text { points) }\end{array}$} & \multicolumn{6}{|c|}{ Educational Level } & \multirow{2}{*}{\multicolumn{2}{|c|}{ Total }} & \multirow{3}{*}{$\begin{array}{c}\text { Quality } \\
\text { level }\end{array}$} \\
\hline & \multicolumn{2}{|c|}{ Early childhood } & \multicolumn{2}{|c|}{ Primary } & \multicolumn{2}{|c|}{ Secondary } & & & \\
\hline & amount & $\%$ & amount & $\%$ & amount & $\%$ & amount & $\%$ & \\
\hline $14-16$ & 76 & 38.58 & 271 & 38.28 & 363 & 39.24 & 710 & $\begin{array}{c}38.80 \\
(2)\end{array}$ & highest \\
\hline $11-13$ & 93 & \begin{tabular}{|c|}
47.21 \\
$(1)$
\end{tabular} & 302 & $\begin{array}{l}42.66 \\
\text { (3) }\end{array}$ & 424 & $\begin{array}{c}45.84 \\
(2)\end{array}$ & 819 & $\begin{array}{l}44.76 \\
\text { (1) }\end{array}$ & high \\
\hline 8- 10 & 28 & 14.22 & 135 & 19.06 & 138 & 14.92 & 301 & $\begin{array}{c}16.45 \\
(3)\end{array}$ & fair \\
\hline Total & 197 & 100 & 708 & 100 & 925 & 100 & 1,830 & 100 & \\
\hline
\end{tabular}

6. Early childhood, primary, and secondary students' innovative skills were at a high level mostly; 45.69 percent, then 33.34 percent were at the highest level. Consideration of each education level found that primary students' innovative skills were at the high level; 49.87 percent, followed by early childhood students at 44.17 percent, and secondary students at 42.81 percent.

Table 6 showsstudents' innovative skills

\begin{tabular}{|c|c|c|c|c|c|c|c|c|c|}
\hline \multirow{3}{*}{$\begin{array}{c}\text { Score range } \\
\text { (total 20 } \\
\text { points) }\end{array}$} & \multicolumn{6}{|c|}{ Educational Level } & \multirow{2}{*}{\multicolumn{2}{|c|}{ Total }} & \multirow{3}{*}{$\begin{array}{l}\text { Quality } \\
\text { level }\end{array}$} \\
\hline & \multicolumn{2}{|c|}{ Early childhood } & \multicolumn{2}{|c|}{ Primary } & \multicolumn{2}{|c|}{ Secondary } & & & \\
\hline & amount & $\%$ & amount & $\%$ & amount & $\%$ & amount & $\%$ & \\
\hline $18-20$ & 68 & 34.52 & 227 & 32.06 & 315 & 34.05 & 610 & $\begin{array}{c}33.34 \\
(2)\end{array}$ & highest \\
\hline $14-17$ & 87 & $\begin{array}{c}44.17 \\
(4)\end{array}$ & 353 & $\begin{array}{c}49.87 \\
\text { (1) }\end{array}$ & 396 & $\begin{array}{c}42.81 \\
(3) \\
\end{array}$ & 836 & $\begin{array}{c}45.69 \\
\text { (1) }\end{array}$ & high \\
\hline $10-13$ & 42 & 21.32 & 128 & 18.07 & 214 & 23.14 & 384 & $\begin{array}{c}20.99 \\
\text { (3) }\end{array}$ & fair \\
\hline Total & 197 & 100 & 708 & 100 & 925 & 100 & 1,830 & 100 & \\
\hline
\end{tabular}

7. Early childhood, primary, and secondary students' innovative construction skills were ata high

level $(=2.16$, S.D $=0.33)$ mostly. Consideration of each education level found that primary students had innovative construction skills were at a high level $(=2.26, \mathrm{~S} . \mathrm{D}=0.28)$, then secondary students $(=2.11$, S.D $=0.36)$, and early childhood students $(=2.09, \mathrm{~S} . \mathrm{D}=0.35)$.

Table 7 showsstudents' innovative construction skills

\begin{tabular}{|c|c|c|c|c|c|c|c|c|c|c|c|c|}
\hline \multirow[b]{3}{*}{$\begin{array}{l}\text { Item for } \\
\text { evaluation }\end{array}$} & \multicolumn{9}{|c|}{ Educational Level } & \multirow{2}{*}{\multicolumn{3}{|c|}{ Total }} \\
\hline & \multicolumn{3}{|c|}{ Early childhood } & \multicolumn{3}{|c|}{ Primary } & \multicolumn{3}{|c|}{ Secondary } & & & \\
\hline & $\overline{\mathrm{X}}$ & S.D & 遷 & $\overline{\mathrm{X}}$ & S.D & 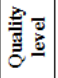 & $\overline{\mathrm{X}}$ & S.D & 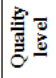 & $\overline{\mathrm{X}}$ & S.D & 这 \\
\hline $\begin{array}{c}1 . \\
\text { Innovativeness }\end{array}$ & $\begin{array}{l}2.07 \\
(3)\end{array}$ & 0.45 & high & $\begin{array}{c}2.31 \\
(3)\end{array}$ & 0.42 & high & $\begin{array}{c}2.01 \\
(6)\end{array}$ & 0.38 & high & $\begin{array}{c}2.13 \\
(4)\end{array}$ & 0.41 & high \\
\hline $\begin{array}{l}\text { 2. Goal and } \\
\text { objective of } \\
\text { innovation } \\
\text { development }\end{array}$ & $\begin{array}{l}2.29 \\
(1)\end{array}$ & 0.39 & high & $\begin{array}{c}2.03 \\
(6)\end{array}$ & 0.22 & high & $\begin{array}{c}2.31 \\
(1)\end{array}$ & 0.44 & high & $\begin{array}{c}2.21 \\
(1)\end{array}$ & 0.35 & high \\
\hline $\begin{array}{l}\text { 3. Use of } \\
\text { knowledge for } \\
\text { developing } \\
\text { innovation }\end{array}$ & $\begin{array}{l}2.14 \\
(2)\end{array}$ & 0.24 & high & $\begin{array}{c}2.32 \\
(2)\end{array}$ & 0.28 & high & $\begin{array}{c}2.10 \\
(3)\end{array}$ & 0.26 & high & $\begin{array}{c}2.18 \\
(2)\end{array}$ & 0.26 & high \\
\hline $\begin{array}{l}\text { 4. Innovative } \\
\text { design }\end{array}$ & $\begin{array}{l}2.00 \\
(4)\end{array}$ & 0.29 & high & $\begin{array}{c}2.46 \\
(1)\end{array}$ & 0.41 & high & $\begin{array}{c}2.05 \\
(5)\end{array}$ & 0.26 & high & $\begin{array}{c}2.17 \\
(3)\end{array}$ & 0.32 & high \\
\hline $\begin{array}{l}\text { 5. Procedures } \\
\text { of innovative } \\
\text { development }\end{array}$ & $\begin{array}{c}2.00 \\
(4)\end{array}$ & 0.29 & high & $\begin{array}{c}2.25 \\
(4)\end{array}$ & 0.18 & high & $\begin{array}{c}2.07 \\
(4)\end{array}$ & 0.37 & high & $\begin{array}{c}2.10 \\
(6)\end{array}$ & 0.28 & high \\
\hline $\begin{array}{l}\text { 6. Success of } \\
\text { innovative } \\
\text { development }\end{array}$ & $\begin{array}{c}2.07 \\
(3)\end{array}$ & 0.45 & high & $\begin{array}{c}2.16 \\
(5)\end{array}$ & 0.18 & high & $\begin{array}{c}2.12 \\
(2)\end{array}$ & 0.44 & high & $\begin{array}{c}2.11 \\
(5)\end{array}$ & 0.35 & high \\
\hline Total & $\begin{array}{l}2.09 \\
(3)\end{array}$ & 0.35 & high & $\begin{array}{c}2.26 \\
(1) \\
\end{array}$ & 0.28 & high & \begin{tabular}{|c|}
2.11 \\
$(2)$
\end{tabular} & 0.36 & high & 2.16 & 0.33 & high \\
\hline
\end{tabular}


8. Early childhood, primary, and secondary students' morality and ethics were at a high level ( $=4.43$, S.D $=0.51$ ).

Table 8 showsstudents' morality and ethics

\begin{tabular}{|c|c|c|c|c|c|c|c|c|c|c|c|c|c|}
\hline \multirow[b]{3}{*}{ items } & \multirow{3}{*}{$\begin{array}{l}\text { Item for } \\
\text { evaluation }\end{array}$} & \multicolumn{9}{|c|}{ Educational Level } & \multirow{2}{*}{\multicolumn{3}{|c|}{ Total }} \\
\hline & & \multicolumn{3}{|c|}{ Early childhood } & \multicolumn{3}{|c|}{ Early childhood } & \multicolumn{3}{|c|}{ Early childhood } & & & \\
\hline & & $\overline{\mathrm{x}}$ & S.D & 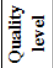 & $\overline{\mathrm{X}}$ & S.D & 逽 & $\overline{\mathrm{x}}$ & S.D & 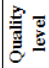 & $\overline{\mathrm{X}}$ & S.D & 列 \\
\hline 1 & $\begin{array}{l}\text { Have desirable } \\
\text { characteristics }\end{array}$ & $\begin{array}{c}4.42 \\
(1)\end{array}$ & 0.57 & high & $\begin{array}{c}4.64 \\
(1)\end{array}$ & 0.44 & highest & $\begin{array}{c}4.41 \\
(1)\end{array}$ & 0.43 & high & $\begin{array}{c}4.49 \\
(1)\end{array}$ & 0.48 & high \\
\hline 2 & $\begin{array}{l}\text { Help each } \\
\text { otherand be } \\
\text { grateful }\end{array}$ & $\begin{array}{c}4.16 \\
(3)\end{array}$ & 0.52 & high & $\begin{array}{c}4.60 \\
(2)\end{array}$ & 0.49 & highest & $\begin{array}{l}4.15 \\
(4)\end{array}$ & 0.46 & high & $\begin{array}{l}4.30( \\
4)\end{array}$ & 0.49 & high \\
\hline 3 & $\begin{array}{l}\text { Accept } \\
\text { different } \\
\text { though and } \\
\text { culture }\end{array}$ & $\begin{array}{c}4.41 \\
(2)\end{array}$ & 0.53 & high & $\begin{array}{c}4.64 \\
(1)\end{array}$ & 0.46 & highest & $\begin{array}{c}4.36 \\
(2)\end{array}$ & 0.51 & high & $\begin{array}{l}4.47 \\
(2)\end{array}$ & 0.50 & high \\
\hline 4 & $\begin{array}{l}\text { Realize, } \\
\text { appreciate the } \\
\text { valueof } \\
\text { environment } \\
\text { reservation and } \\
\text { development }\end{array}$ & $\begin{array}{c}4.14 \\
(4)\end{array}$ & 0.54 & high & $\begin{array}{c}4.57 \\
(4)\end{array}$ & 0.44 & highest & $\begin{array}{l}4.24 \\
(3)\end{array}$ & 0.54 & high & $\begin{array}{c}4.31 \\
(3)\end{array}$ & 0.50 & high \\
\hline & Total & $\begin{array}{c}4.33 \\
(3)\end{array}$ & 0.56 & high & $\begin{array}{c}4.62 \\
(1)\end{array}$ & 0.46 & highest & $\begin{array}{c}4.34 \\
(2)\end{array}$ & 0.49 & high & 4.43 & 0.51 & high \\
\hline
\end{tabular}

Early childhood, primary, and secondary students' opinion toward GPAS 5 Steps instruction

were at a high levelpositively.

Part 3: The dissemination of GPAS 5 Steps teaching model for enhancing learning skills in Thailand 4.0 era

1. Instructors aspect found that: 1.1) early childhood, primary, and secondary instructors' knowledge and understanding about GPAS 5 Steps were at a high level in aspects of knowledge, skill, and attitude. And 1.2) instructors' teaching ability according to GPAS 5 steps were at high level in aspects of instructor, learning activities, learning materials and supported resources, assessment and evaluation along with classroom atmosphere and management.

2. Students aspect found that 2.1) students' higher order thinking were at a high level; analytical thinking, evaluation, and creativity, 2.2) innovative skills were at a high level, 2.3) innovative construction skills were at a high level, 2.4) students' behaviors that indicated their morality, ethics, and expectations of social value were at a high level, and 2.5) students' opinion toward GPAS 5 Steps instruction found that instructional procedures, classroom atmosphere, and evaluation were at high levels.

3. Experts' opinions toward the use of GPAS 5 Steps teaching model in 4 regions found that:

3.1 The GPAS 5 Steps teaching model for enhancing learning skills in Thailand 4.0 era in

school revealed that PLC members met via online and faceto-face for setting mission objectives and goals, sharing knowledge from head of instructors and school administrators, using logbook for driving PLC processes, and lesson studying.

3.2 TheGPAS 5 Steps teaching model for enhancing learning skills in Thailand 4.0 era in

schoolprovided benefit for instructors including portfolio creationfor further classroom research, integrating various subjects, and practicing open-ended class questions.

3.3 Initially,use of GPAS empowered students to work collaboratively but also make

reflective independent decisions about pedagogy and the appropriate use of materials and class resources.

\section{Discussion}

1. Knowledge and understanding of GPAS early childhood, primary, and secondary instructors were at a consistently high level and fitted functionally into theresearch hypothesis because of the development of GPAS 5 Steps exalted teaching model for enhancing learning skills in Thailand 4.0 era used knowledge sharing as a second step of the procedure. The coaches and experts of the Institute of Academic Development (AID) (2018) provided a chance to school administrators and instructors'knowledge for sharing GPAS 5 steps instruction. Furthermore, it provided opportunitiesto presentexamples and instructional innovation that related to instruction, technology materials, and authentic assessment and evaluation, and performance ability evaluation. According to Richard Dufour and Eaker (1998) ensuring that students learn and accept new choices are basic tenets of PLC which not only clarify task performance but also pinpoints the areas where students need to improve.

This approach is consistent with SowimonSaphuksri (2019) data about the professional learning community which blended technologies to enhance the competency of learning management by art-based education to improve the skills of creative innovation for elementary students; the results suggest that after using the PLC model, instructors displayed greater knowledge and understanding thanin previous class observations, around .05 level of statistical significance.

2. Instructional design ability accorded to GAPS 5 Steps of early childhood, primary, and secondary instructors were at high level mostly, consistency with research hypothesis 1.2 because of GPAS 5 Steps exalted teaching model for enhancing learning skills in Thailand 4.0 era used knowledge sharing as a second steps of the procedures. Hord (1997: 14-24) stated that the professional learning community (PLC) was the process of working collaboratively which enhancedcreative ability, a concept consistent with McMilan and Chavis (1986) who claimed that knowledge sharing provided opportunities for sharing ideas, thinking, emotions, and information through PLC processes.

3. Instructional ability accorded to GAPS 5 Steps of early childhood, primary, and secondary instructors were at the highest level mostly; 69.42 percent, consistency with research hypothesis 1.3 because of GPAS 5 Steps exalted teaching model for enhancing learning skills in Thailand 4.0 era used lesson study as a forth steps of the procedures. School administrators, instructors, coaches, and experts joined collaboratively within online and face-to-facePLC processes of each learning subject areas in order to communicate, share, supervise, coach, observe, and monitor the operation. Thus, all participants worked collaboratively to set the classroom observation schedules; 2-3 times, then gave reflective feedback toward instruction and students' behavior after the observation. The lesson study consisted with 3 sub-activities; 1) plan and design lesson, 2) implement and observe, and 3) review and refine for improvement. First, plan and design lesson stage, according to DuFour and Eaker (1998) suggesting that PLC was the action orientation; all members emphasized on bringing principle to use and do in the real situations. Likewise, 
Stigler and Hiebert (1999) stated that lesson study for development relied on attemptof all members, which used cautious observation and cycle collaborative working were identified the tools for instructional development in order to reach the learning students' goal.

4. Innovative construction skills of early childhood, primary, and secondary instructors were at high level mostly; 58.83 percent, consistency with research hypothesis 1.4 , because coaches of early childhood, primary, and secondary coaches of Silpakorn Demonstration School brought examples of curriculum, instruction, assessment and evaluation and technology innovation and research for instructors to share knowledge about various types of educational innovation. According to WasanSutthawart and PhitakSiriwong (2015) the innovation for development gave extreme benefits to the education system. According to JirapornRodpuang (2016) observations of a professional learning community model to enhance the skills of learning management, systems thinking and creative innovation of student teachers, the results suggestedthat the average score for learning management, systems thinking and creative innovation skills, were first, second, third and fourth, overall, after the trial with the Professional Learning Community Model of student teachers increased significantly at a level of .05 level, which complied with the effectiveness criteria of the model had been defined.

5. Higher order thinking of early childhood, primary, and secondary students were in high level mostly; 44.76 percent, consistency with research hypothesis 2.1. Because of GPAS 5 steps instruction was a systematic instruction (Institute of Academic. Development (AID) ,2018) which developed learners to think, act, and solve problems, consistency with Ministry of Education (2018) identifiedstudent-centered learning procedures in The Basic Education Core Curriculum B.E. 2551 (A.D. 2008) that learners used variety ways for learning.

6. Innovative skills of early childhood, primary, and secondary students were at high level mostly; 45.69 percent, which is consistent with research hypothesis 2.2 , because GPAS 5 Steps was systematic instruction which emphasized on 5 steps of thinking (Institute of Academic Development (AID), 2018). Gathering and processing were the learning procedures for enhance both basic and higher order thinking of students. The learning goal focused on the cognitive ability of learners to think, act, and solve problem. According to Bagley (2014) the innovator was the person who stated, found, learnt, and act with the new way, also think and act differently. Conformity with the 20-Year National Strategy (2017-2036) (Office of the Education Council, 2017) identified learner aspirations that developed all students in order to have characters and learning skills (3Rs8Cs)related to 21 st century era. And students also improved their potentials and learnt continuously for being skillful and having competency to respond employment, national development, and dynamic change in 21 st century and 4.0 economy and society era.

7. Innovative construction ability of early childhood, primary, and secondary students were at high level mostly, consistency with research hypothesis 2.3 , because the GPAS 5 Steps instruction were taught systematically for students (Institute of Academic Development (AID), 2018), especially learning and innovative skills that useful for instruction in Thailand 4.0 era. According to strategy and planinvolved principles of education that using science and technology for enhancing, researching, innovation of development. This is consistent with the eighth policy of 20Year National Strategy (2017-2036) (Office of the National Economics and Social Development Council, 2018)which mentioned that development and enhancement of using science and technology for research and innovation development. In addition, the 20-Year National Strategy (2017-2036) (Office of the Education Council, 2017) argued that learner aspirations must develop their 21st century potential and learning skills (3Rs8Cs), continuously improve and self-directly learn in order to have skills and competency to respond employment, national development, and dynamic change in 21 st century and 4.0 economy and society era.

8. Morality and ethics of early childhood, primary, and secondary students were at high level mostly, consistency with research hypothesis 2.4 , because of providing GPAS 5 Steps instruction. The instruction emphasized on team working, students therefore had to work together, especially self-regulating evaluation that focused on morality and ethics of students (Institute of Academic Development (AID), 2018). Hence, consistency with the 20-Year National Strategy (2017-2036) (Office of the National Economics and Social Development Council, 2017) mentioned that morality, ethics, social value, and consciousness were nurtured through education, and national education policy, strategy and that future planning must nurture good economic value and a social and cultural learning consciousness. A fifth strategy of Education National Strategy (2017-2036) (Office of the Education Council, 2017) suggested that education enhanced quality of life by having morality, ethics and using a sufficiency economy. Moreover, the Ministry of Education (2018) in The Basic Education Core Curriculum B.E. 2551 (A.D. 2008)contended that morality, ethics, desired value, selfesteem, discipline, religious perspectives and a sufficiency economy should underpin learner goals in the 21 st century curriculum.

\section{Suggestions}

\section{Suggestion for using research results}

\subsection{For school administrators}

1) School administrators, instructors, and stakeholders need to endeavor to graspthe components

of model and monitor as evaluation to all steps of using model as well.

2) School administrators should consider all factors to use the model successfully.

1.2. For instructors

1) Using model with variety technology for all steps of PLC.

2) Collaborative clarifying is important for using GPAS 5 instruction that affects on students learning quality. 


\section{Suggestions for further research}

1) Should develop exalted teaching model for enhancing others learning skills such as online instruction.

2) Should develop GPAS 5 Steps exalted teaching model for enhancing other skills such as digital literacy, future skills, or needed 21 st century skills of students continuously.

\section{References}

[1] Bagley, Rebecca O. (2014). The 10 Traits of Great Innovators. Retrieved from http://www.\#1046058ed50b

[2] DuFour and Eaker. (1998). Professional Learning Communities at Work: Best Practices for Enhancing Student Achievement. Bloomington, IN: National Educational Service.

[3] Hord. (1997). Professional learning communities: Communities of continuous inquiry and improvement. Texas: Southwest Educational Development Laboratory.

[4] McMilan and Chavis. (1986). Sense of community: A definition and theory. American Journal of Community Psychology, 14(1), 6-23.

[5] Stigler \& Hiebert. (1999). Teaching gap: Best ideas from the world's teachers for improving Education in the classroom. New York: Free Press. forbes.com/sites/rebeccabagley/2014/01/1 5/the-10-traits-of-great-innovators/

Translated references:

[6] Institute of Academic Development (AID). (2018). GPAS 5 Steps. Bangkok: Institute of Academic Development company, Ltd.

[7] JirapornRodpuang. (2016). The development of a professional learning community model to enhance the skills of learning management, systems thinking and creative innovation of student teachers. Veridian E-Journal, Silpakorn University 10, 1: January-April.

[8] Ministry of Education. (2018). Basic Education Core Curriculum B.E. 2551 (edition B.E. 2560). Accessed on
September 25, 2018 from http://academic.obec.go.th/newsdetail.php ?id $=75$

[9] Office of the Education Council, Ministry of Education. (2017). National Education Plan 2017-2036. Bangkok: Prikwan Graphic Press.

[10] Office of the Education Council, Ministry of Education. (2018). National Education Standard 2017. Accessed on September 19, 2017.2 From http://www.http://qa.vru.ac.th/

[11] Office of the National Economics and Social Development Council. (2018). 20Year National Strategy (2017-2036). Accessed on September 17, 2018. From http://www.nesdb.go.th

[12] SiriwanVanichwatanavorachai. (2016). Differentiated Instruction. Education Journal 13, 2: November 2015-March 2016.

[13] SuwimonSaphuksri. (2019). "Professional Learning Community Blended Technologies To Enhance The Competency Of Learning Management By Art Based Education And Improve The Skills Of Creative Innovation Creation For Elementary Students." Dissertation of Doctor of Philosophy Education, Silpakorn University.

[14] WasanSutthawart and PhitakSiriwong. (2015). The Basic Educational Innovator in Public Sector: A Study for Grounded Theory. Veridian E-Journal, Silpakorn University 8, 2: May-August. 